\title{
3D PRINTED HYDROGEL GLUCOSE SENSOR ON ARGON PLASMA ACTIVATED POLYSTYRENE
}

\author{
Nenad Krstic ${ }^{1,2^{*}}$, Jens Jüttner ${ }^{1^{*}}$, Achim Müller ${ }^{3}$, Monika Knuth $^{3}$, \\ Christiane Thielemann ${ }^{1}$
}

\author{
${ }^{1}$ BioMEMS Lab, Faculty of Engineering, Technische Hochschule Aschaffenburg, Würzburger Str. 45, \\ 63743 Aschaffenburg, Germany \\ 2Department of Chemistry, Faculty of Science and Mathematics, University of Nis, Visegradska 33, \\ 18106 Nis, Serbia
}

${ }^{3}$ EyeSense GmbH, Stockstädter Straße 17, 63762 Großostheim, Germany

*Authors contributed equally

\begin{abstract}
This study presents a proof of principle concept for a two-dimensional bioprinted glucose sensor on Petri dishes that allows for glucose measurements in cell culture medium. To improve bioink adhesion, the polystyrene surfaces of standard Petri dishes are activated with argon plasma, which increases roughness and hydrophilicity. The bioink containing the sensor chemistry-namely fluorescently labeled ConA/Dextran embedded in alginate microbeads—was printed on the activated Petri dishes with an extrusion-based bioprinter. The printed sensor showed good stability and adhesive properties on polystyrene. The glucose concentration was examined using a standard fluorescence microscope with filters adapted to the emission wavelength of the donor and reference dyes. The printed glucose sensor showed high sensitivity and good linearity in a physiologically relevant range of glucose concentrations.
\end{abstract}

\section{Keywords}

bioprinted sensor, adhesion, Ar plasma, activated polystyrene, glucose measurement

\section{Introduction}

The field of tissue engineering aims to create two-dimensional (2D) and three-dimensional (3D) artificial cellular tissue for regenerative medicine and organ-on-chip models. Generally, 3D culture models improve the physiological relevance and predictive power of the cell-based assays for experiments including application of drugs. An even more important argument to use 3D models is their excellent longevity, well suited for experiments with focus on late developing effects. However, one of the current limiting factors is the nutrient supply of the artificial tissue as necrotic regions may easily develop due to poor nutrient transport.

The most important nutrient for cell cultures is glucose, a component of all common cell culture media [1]. For the control of cell viability, the monitoring of the glucose concentration and gradient within the medium and also (ideally) inside the 3D culture itself is of upmost importance. Many different types of glucose sensors have been investigated and described in literature, mostly based on optical or enzyme-based electrochemical transducer principles [2, 3]. With these sensors, glucose has been measured in blood [4, 5], in tear fluid [6, 7], in physiological fluids [5, 8] and even through skin $[9,10]$. Glucose sensors for cell culture medium have been proposed [11, 12], typically based on bulky electrochemical transducers, not suitable for a sensing resolution in the millimeter range.

A very promising enzyme-free sensor chemistry for detection of glucose was developed, initially intended for continuous glucose measurement for diabetes patients [1]. This approach of detecting glucose is based on a competitive complex formation between Concanavalin (ConA) and dextran. The receptor ConA and the competitor dextran (in the following called "sensor chemistry") are labelled with fluorescent dyes, which, in the case of joint complex formation, exhibit resonance-based quenching of their fluorescence (Fluorescence resonance energy transfer, FRET). The 
displacement of dextran by glucose leads to an increased distance between the two fluorophores (donor dye on dextran and acceptor dye on ConA), which prevents the non-radiative energy transfer of the FRETeffect. Therefore, the dyed dextran is again able to emit a certain intensity of light which is used as indicator for the glucose concentration. This principle is reversible when glucose concentration decreases, and it can be measured with appropriate fluorescence photometers. Furthermore, a third dye can be used as a marker to provide a reference point during measurement [13-15]. This sensor chemistry is embedded in a hydrogel, which can be stored for many months in a dried state.

Based on this optical transducer principle, we present a proof of principle concept for a bioprinted glucose sensor with good lateral resolution. The described dextran/ConA sensor chemistry embedded in a hydrogel shall be bioprinted onto standard cell culture dishes and shall be used as a glucose sensor for cell culture. The bioprinting process places a large number of demands on the "bioink" used, such as printability, dimensional stability and biocompatibility. However, preliminary experiments showed that adhesion properties of the bioprinted sensor are the most urgent challenge. This is due to the nature of the printed hydrogel and the properties of the culture dish surface made of hydrophobic polystyrene (PS). To achieve better adhesion between printed bioink and the PS, standard Petri dishes were modified with Argon (Ar) plasma. The glucose sensor was fabricated by printing hydrogel directly onto the Petri dish surface and was tested for its ability to determine glucose in cell media.

\section{Materials and Methods}

\section{Plasma modification of the polystyrene Petri dish surface}

With the aim to change the surface properties (hydrophobic / hydrophilic) of the PS Petri dish, its surface was treated with Ar plasma with a LFG 40 plasma generator (Diener electronic GmbH + Co. KG, Ebhausen, Germany) under the following conditions: SP: 9 sccm, CV: 9 sccm, 0.300 mbar, temperature: 26$28{ }^{\circ} \mathrm{C}$ and time of treatment: $10 \mathrm{~min}$. For this experiment transparent PS Petri dishes $(35 \times 10 \mathrm{~mm})$ (Sarstedt, Nümbrecht, Germany) were used. The Petri dishes were pre-treated with the SARSTEDT standard surface for adherent cells which introduces hydrophilic groups into the PS (in the following called "untreated asorigin”).

Changes in the surface properties of PS were characterized via water contact angle measurements with a Drop Shape Analyzer DSA25 (KRÜSS GmbH, Hamburg, Germany) and AFM characterization using a MFP-3D-BIO AFM (Asylum Research, Oxford Instruments, Santa Barbara, California, USA).

\section{Bioink Sensor Chemistry}

The proprietary hydrogel formulation contains fluorescently labeled ConA and Dextran (sensor chemistry) loaded to previously formed alginate microbeads by incubation of the beads in a solution of $4 \mathrm{mg} / \mathrm{ml}$ ConA and $7 \mathrm{mg} / \mathrm{ml}$ Dextran. Alginate microbeads were mixed with an ultraviolet-polymerizable, derivatized poly(vinyl alcohol). The reference dye was also embedded into microbeads. The highly viscous solution is processed as bioink using a bioprinter. This printable bioink was provided by the coauthor EyeSense $\mathrm{GmbH}$ (Grossostheim, Germany) [1, 14].

\section{D Sensor Printing}

The bioink was printed on Petri dishes with an extrusion-based bioprinter 3DDiscovery ${ }^{\mathrm{TM}}$ (regenHU, Villaz-Saint-Pierre, Switzerland), under the following conditions: $0.41 \mathrm{~mm}$ dosing needle, $2 \mathrm{~mm} / \mathrm{s}$ feed rate and 2.5 bar process pressure. The shape of the printed hydrogel sensor is shown in Figure 1. Afterwards, printed hydrogel was cured with UV M300L4-300 nm, $26 \mathrm{~mW}$ (Min) Mounted LED, $350 \mathrm{~mA}$ lamp (Thorlabs $\mathrm{GmbH}$, Dachau, Germany) for $60 \mathrm{~s}+60 \mathrm{~s}$ at room temperature to gain final form stability. After UV curing, the system was washed with demi- $\mathrm{H}_{2} \mathrm{O}$ with the aim to remove non cross-linked material from printed sensor hydrogel.

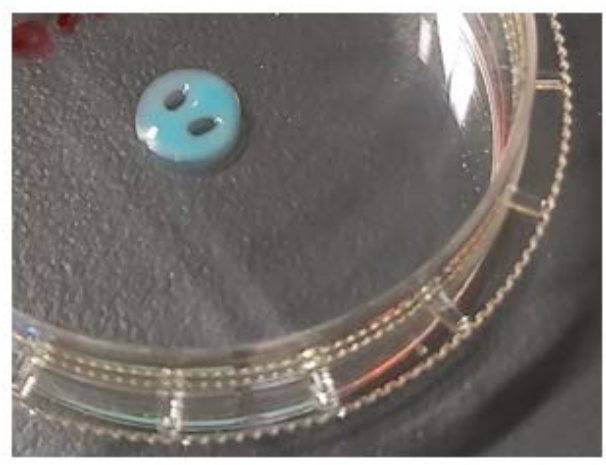

Fig. 1: 3D printed sensor hydrogel on Ar plasma activated Petri dish.

\section{Glucose Imaging Setup}

3D printed hydrogel glucose sensors in plasma activated Petri dishes were exposed to $2 \mathrm{~mL}$ of RPMI cell culture medium (Sigma-Aldrich, St. Louis, MO, USA) containing different concentrations of glucose (0-5 g/L) for 30 min each. The varying concentrations were realized by mixing glucose-free RPMI medium with the corresponding amount of $20 \%$ glucose stock solution. For every concentration, samples were examined using a fluorescence microscope Nikon Eclipse Ti (Minato, Tokyo, Japan) with fluorescence color filters for the emission of the donor dye (bandpass: $620 \mathrm{~nm}$ ) and for the emission of the reference dye (bandpass: $750 \mathrm{~nm})$. The excitation wavelength emitted by a mer- 
cury lamp was restricted to a wavelength of $590 \mathrm{~nm}$ due to an excitation filter (bandpass: $590 \mathrm{~nm}$ ).

\section{Image Analysis and Value Formation}

A custom-made MATLAB-tool was used for image analysis. Firstly, fluorescence calibration images of the Petri dish were subtracted from 16-bit greyscale images of each color filter (donor and reference). Afterwards, the quotient donor/reference of both summed up pixel intensities was determined. This value is an indicator for the concentration of glucose in media [16].

\section{Results}

Results of contact angle (CA) measurement and AFM characterization of untreated and argon plasma treated PS Petri dishes are presented in Table 1.

Table 1: Contact angles and AFM data.

\begin{tabular}{lc|cc}
\hline & CA & \multicolumn{2}{|c}{ AFM } \\
\cline { 2 - 4 } & $\left.\mathbf{(}^{\circ}\right)$ & $\mathbf{R}_{\mathbf{q}}(\mathbf{n m})$ & $\mathbf{R}_{\mathbf{a}}(\mathbf{n m})$ \\
\hline untreated PS & 67.77 & 3.093 & 2.517 \\
treated PS & 45.98 & 4.390 & 3.585 \\
\hline
\end{tabular}

Fluorescence images are shown exemplarily in Figure 2, while the sensor response curve for glucose determination in cell culture medium in the concentration range 0 to $5 \mathrm{~g} / \mathrm{L}$ is shown in Figure 3.

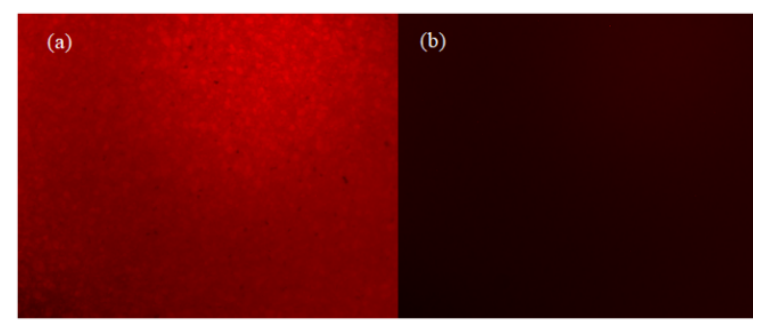

Fig. 2: Fluorescence-response of a printed sensor at $5 \mathrm{~g} / \mathrm{L}:$ a) donor signal and b) reference signal.

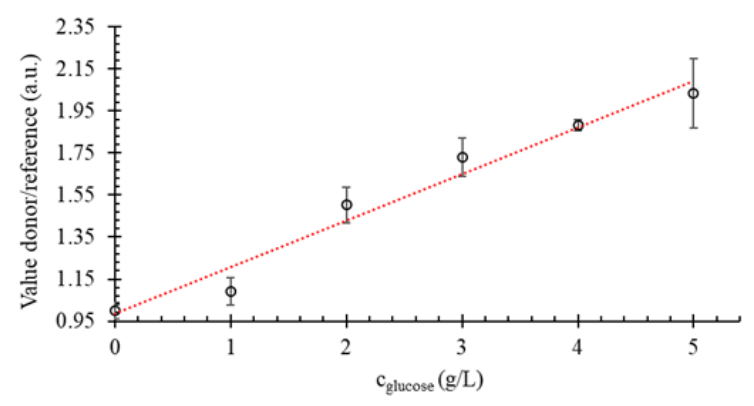

Fig. 3: Sensor response curve for glucose determination in RPMI cell culture medium.

\section{Discussion}

Using Ar plasma as a non-invasive method, we achieved the modification of the polymeric surface properties of Petri dishes without affecting the bulk of the material. When inert gas is used to form the plasma, free radicals will be created onto the surface and can be subsequently used to form functional groups which change the properties of the treated surface [17]. Results of water contact angle and AFM measurements indicate the hydrophilic transformation of the polymer surfaces. Measured contact angles of untreated and treated PS samples were $67.77^{\circ}$ and $45.98^{\circ}$, respectively (Table 1). Obtained AFM data root mean square (RMS) of roughness $R_{q}(n m)$ and mean roughness $R_{a}$ $(\mathrm{nm})$ are 3.093 and 2.517 for untreated as-origin PS, while for Ar plasma modified PS their values are increased slightly (4.390 and 3.585). These results reveal the improvement of PS wettability after $\mathrm{Ar}$ plasma treatment which is in accordance to literature data [18-20]. Stability of printed sensor hydrogel (Fig. 1) on the Ar plasma activated PS surface was investigated for one week in cell culture medium (RPMI), followed by one week in demi-water. During this period, the sensor hydrogel was stable and showed good adhesion on the activated PS surface.

For the analysis of the printed glucose sensor, a physiologically relevant range ( 0 to $5 \mathrm{~g} / \mathrm{L}$ ) of glucose in cell medium was chosen (Fig. 3). Fluorescence amplitudes were captured (three different points on printed sensor hydrogel for each concentration), as shown exemplarily in Figure 2, and analyzed as described above. Mean values and standard deviation of the values (donor/reference) obtained with the custommade MATLAB tool were calculated and afterwards this dataset was normalized to the reference at $0 \mathrm{~g} / \mathrm{L}$ glucose. The resulting sensor response in RPMI cell medium is shown in Figure 3.

According to the data from Fig. 3, the biosensor shows high sensitivity and good line correlation (coefficient of determination $\mathrm{R}^{2}=0.9681$ ) under the investigated conditions. These results are in accordance with the data obtained in our previous study [16].

\section{Conclusion}

In this study we successfully modified the PS surface of a Petri dish with Ar plasma to improve its hydrophilicity. As a result, we are able to print glucose sensing molecules embedded in a hydrogel on the activated surface allowing for stable measurements in glucose containing cell culture medium. The analysis of the fluorescent sensor signal has been performed with a standard inverse microscope, allowing to easily transfer this method of glucose measurement to other working groups. The printed sensor showed good 
stability and adhesive properties for at least two weeks in medium. Its glucose response curve in cell media revealed high sensitivity and good linearity under the investigated experimental conditions.

In future work the properties of the sensor hydrogel will be modified in terms of improved bioprintability with the goal to print a well-defined 2D grid allowing for laterally resolved glucose measurements.

\section{Acknowledgement}

This work was funded by the project GlukZ (AZ1253-16) and 3DGlukZ (PDOK-93-19) of the Bavarian Research Foundation. We also thank M. Eng. S. Allig for conducting preliminary experiments.

\section{References}

[1] Müller AJ, Knuth M, Nikolaus KS, Krivánek R, Küster F, Hasslacher C, Auffarth GU. Blood glucose self-monitoring with a long-term subconjunctival glucose sensor. Journal of Diabetes Science and Technology, Jan 2013;7(1):24 34. DOI: $\underline{10.1177 / 193229681300700104}$

[2] Lee H, Hong YJ, Baik S, Hyeon T, Kim DH. Enzyme-based glucose sensor: from invasive to wearable device. Advanced healthcare materials. 2018 Apr;7(8):1701150. DOI: 10.1002/adhm.201701150

[3] Sharafeldin M, Jones A, Rusling JF. 3D-printed biosensor arrays for medical diagnostics. Micromachines. 2018 Aug;9(8): 394. DOI: $10.3390 / \mathrm{mi} 9080394$

[4] Wang J. Glucose biosensors: 40 years of advances and challenges. Electroanalysis: An International Journal Devoted to Fundamental and Practical Aspects of Electroanalysis. 2001 Aug;13(12):983-8. DOI: 10.1002/15214109(200108)13:12<983::AID-ELAN983>3.0.CO;2-\%23

[5] Makaram P, Owens D, Aceros J. Trends in nanomaterial-based non-invasive diabetes sensing technologies. Diagnostics. 2014 Jun;4(2):27-46. DOI: 10.3390/diagnostics4020027

[6] Badugu R, Lakowicz JR, Geddes CD. Fluorescence sensors for monosaccharides based on the 6-methylquinolinium nucleus and boronic acid moiety: potential application to ophthalmic diagnostics. Talanta. 2005 Feb 15;65(3):762-8. DOI: 10.1016/j.talanta.2004.08.003

[7] Senior M. Novartis signs up for Google smart lens. September 2014;32(9):856. DOI: 10.1038/nbt0914-856

[8] Liu C, Sheng Y, Sun Y, Feng J, Wang S, Zhang J, Xu J, Jiang D. A glucose oxidase-coupled DNAzyme sensor for glucose detection in tears and saliva. Biosensors and Bioelectronics. 2015 Aug 15;70:455-61. DOI: 10.1016/j.bios.2015.03.070

[9] Bandodkar AJ, Wang J. Non-invasive wearable electrochemical sensors: a review. Trends in biotechnology. 2014 Jul 1;32(7):363-71. DOI: 10.1016/j.tibtech.2014.04.005

[10] Bruen D, Delaney C, Florea L, Diamond D. Glucose sensing for diabetes monitoring: recent developments. Sensors. 2017 Aug; 17(8):1866. DOI: $\underline{10.3390 / \mathrm{s} 17081866}$
[11] Tric M, Lederle M, Neuner L, Dolgowjasow I, Wiedemann P, Wölfl S, Werner T. Optical biosensor optimized for continuous in-line glucose monitoring in animal cell culture. Analytical and bioanalytical chemistry. 2017 Sep 1;409(24):5711-21. DOI: 10.1007/s00216-017-0511-7

[12] Olivo J, Foglia L, Casulli MA, Boero C, Carrara S, De Micheli G. Glucose and lactate monitoring in cell cultures with a wireless android interface. In2014 IEEE Biomedical Circuits and Systems Conference (BioCAS) Proceedings 2014 Oct 22 (pp. 400-403). IEEE. DOI: 10.1109/BioCAS.2014.6981747

[13] Ballerstadt R, Schultz JS. A fluorescence affinity hollow fiber sensor for continuous transdermal glucose monitoring. Analytical Chemistry. 2000 Sep 1;72(17):4185-92. DOI: 10.1021/ac000215r

[14] Müller AJ, Knuth M, Nikolaus KS, Herbrechtsmeier P. First clinical evaluation of a new long-term subconjunctival glucose sensor. Journal of diabetes science and technology. $2012 \mathrm{Jul}$; 6(4):875-83. DOI: $10.1177 / 193229681200600419$

[15] Chen L, Hwang E, Zhang J. Fluorescent Nanobiosensors for Sensing Glucose. Sensors. 2018 May;18(5):1440. DOI: 10.3390/s18051440

[16] Jüttner J, Kress L. Functionalized Hydrogel as Glucose Imaging Sensor for in vitro Cell Cultures. International Student Scientific Conference POSTER 2019, Praque, May 23,1-3.

[17] Bitar R, Cools P, De Geyter N, Morent R. Acrylic acid plasma polymerization for biomedical use. Applied Surface Science. 2018 Aug 1;448:168-85. DOI: 10.1016/j.apsusc.2018.04.129

[18] Levif P, Seguin J, Moisan M, Barbeau J. Influence of substrate materials on inactivation of $\mathrm{B}$. atrophaeus endospores in a reduced-pressure argon plasma. Plasma Processes and Polymers, 2011 May 3;8(617-630). DOI 10.1002/ppap.201000212

[19] Guruvenket S, Rao GM, Komath M, Raichur AM. Plasma surface modification of polystyrene and polyethylene. Applied surface science. 2004 Sep 15;236(1-4):278-84. DOI: 10.1016/j.apsusc.2004.04.033

[20] Smirnov AV, Atkin VS, Gorbachev IA, Grebennikov AI, Sinev IV, Simakov VV. Surface modification of polystyrene thin films by RF plasma treatment. BioNanoScience. 2017 Dec 1; 7(4):680-5. DOI: 10.1007/s12668-017-0407-1
Jens Jüttner, B.Eng. BioMEMS Lab

Faculty of Engineering Technische Hochschule Aschaffenburg Würzburger Str. 45 Aschaffenburg, Germany

E-mail:biomems@th-ab.de Phone: +4906021 4206-817 